\title{
Single indium atoms and few-atom indium clusters anchored onto graphene via silicon heteroatoms
}

Kenan Elibol1,2,3,4, Clemens Mangler2, David D. O’Regan3,5, Kimmo Mustonen2, Dominik Eder6, Jannik C. Meyer2,7, Jani Kotakoski2, Richard G. Hobbs3,4, Peter A. van Aken1, Toma Susi2, Bernhard C. Bayer2,6

1. Stuttgart Center for Electron Microscopy, Max Planck Institute for Solid State Research, Heisenbergstr. 1, 70569 Stuttgart, Germany

2. Faculty of Physics, University of Vienna, Boltzmanngasse 5, A-1090, Vienna, Austria

3. Centre for Research on Adaptive Nanostructures and Nanodevices (CRANN) and Advanced Materials and Bio-Engineering Research Centre (AMBER), Dublin 2, Ireland

4. School of Chemistry, Trinity College Dublin, The University of Dublin, Dublin 2, Ireland

5. School of Physics, Trinity College Dublin, The University of Dublin, Dublin 2, Ireland

6. Institute of Materials Chemistry, Vienna University of Technology (TU Wien), Getreidemarkt 9/165, A-1060 Vienna, Austria

7. Institute for Applied Physics, University of Tübingen, Auf der Morgenstelle 10, 72076 Tübingen

* Corresponding author: k.elibol@fkf.mpg.de

We present for the first time the facile self-assembly of single In atoms and few-atom In clusters (2-6 atoms) and their concurrent room-temperature stable anchoring onto individual substitutional Si dopant atoms within graphene [1]. Since single atoms and atomic clusters comprised of only few atoms have distinct physical and chemical properties [2,3], these atomic structures on solid supports attract currently high attention for the potential applications in areas ranging from catalysis to nanoplasmonics [4,5]. Pathways for their controlled fabrication and stable placement remain however scarce. Here, indium was evaporated onto suspended monolayer graphene (that intrinsically includes a small fraction of substitutional Si heteroatoms) using a custom-built preparation chamber (base pressure $\sim 10^{-9}$ mbar) directly coupled to an atomic resolution scanning transmission electron microscope (STEM) [6].

The experiments involving laser irradiation, in situ In deposition, and imaging by STEM are schematically shown in Figure 1a. Our element-sensitive STEM results shown in Figure 1b-f show that the resulting structures incorporates indium clusters anchored onto the graphene lattice via the substitutional Si atoms. These structures appear stable at room temperature under $60 \mathrm{keV}$ electron irradiation. We find that the exact atomic arrangements of these In clusters depend strongly on the original coordination of Si in the graphene lattice. As an example, 3-fold symmetric In clusters form on 3-fold coordinated Si atoms, whereas 4-fold symmetric clusters are found on 4-fold coordinated $\mathrm{Si}$ atoms. In addition, single In atoms and In dimers can also stabilize at Si impurity sites. On the basis of population analysis of semi-local density-functional theory (DFT) calculations, the In clusters anchored on Si are divalent electron donors, where one hole resides on the $\mathrm{Si}$ atom and the 2 nd hole is being shared (not equally unless required by symmetry) by the In atoms. Such artificial, anchored few-atom molecules may find applications for example as few-atom catalysts in heterogeneous catalysis. 


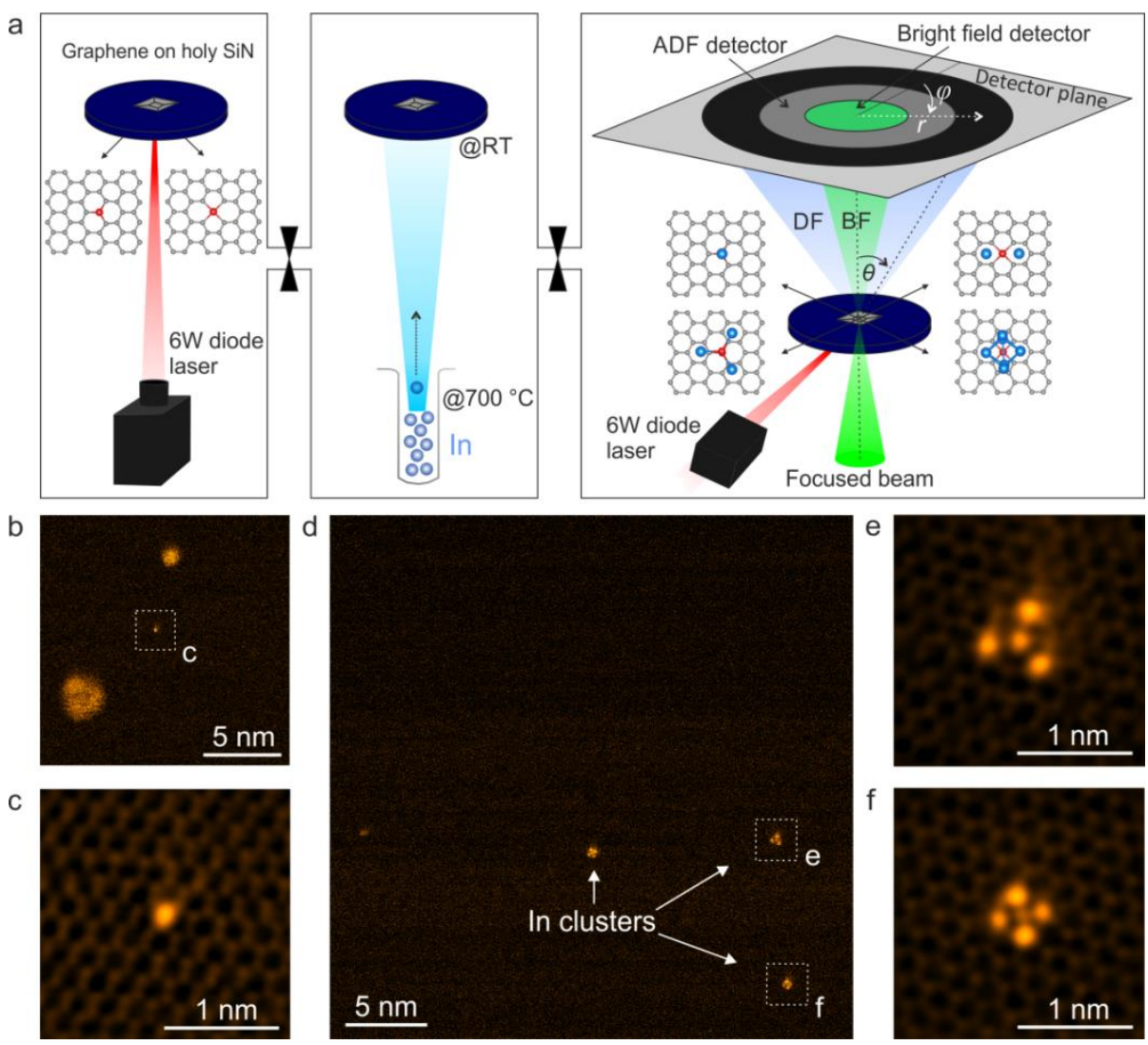

Figure 1. (a) Schematic of the experimental setup used for laser irradiation, in situ In deposition, and imaging by STEM. In, $\mathrm{Si}$ and $\mathrm{C}$ atoms shown on the atomic models are in blue, red and grey, respectively. (b,d) Medium-angle annular dark field (MAADF)-STEM images of a monolayer graphene surface with single In atomand few-atom In clusters. (c,e,f) Close-up MAADF-STEM images of a single In atom and 3-fold and 4-fold symmetric In clusters indicated by white dashed frames in panels b and d. All images display false colour and images in (c,e,f) are double Gaussian filtered after Wiener filtering to reduce noise and enhance contrast.

\section{References}

[1] K. Elibol, C. Mangler, D. D. O'Regan, K. Mustonen, D. Eder, J. C. Meyer, J. Kotakoski, R. G. Hobbs, T. Susi, B. C. Bayer, arXiv:2009.00063 (2020).

[2] N. Vats, Y. Wang, S. Sen, S. Szilagyi, H. Ochner, S. Abb, M. Burghard, W. Sigle, K. Kern, P. A. van Aken, S. Rauschenbach, ACS Nano 14, (2020) 4626-4635.

[3] D. Grandjean, E. Coutiño-Gonzalez, N.T. Cuong, E. Fron, W. Baekelant, S. Aghakhani, P. Schlexer, F. D’Acapito, D. Banerjee, M.B.J. Roeffaers, M.T. Nguyen, J. Hofkens, P. Lievens, Science 361, (2018) 686-690.

[4] Y. Ren, Y. Tang, L. Zhang, X. Liu, L. Li, S. Miao, D. S. Su, A. Wang, J. Li, T. Zhang, Nature Communications 10, (2019) 4500

[5] W. Zhou, J. Lee, J. Nanda, S. T. Pantelides, S. J. Pennycook, J. C. Idrobo, Nature Nanotechnology 7 (2012), 161-165.

[6] K. Elibol, C. Mangler, T. Gupta, G. Zagler, D. Eder, J. C. Meyer, J. Kotakoski, B. C. Bayer, Advanced Functional Materials 30 (2020), 2003300. 